\title{
Cloud Computing, Capabilities and Intercultural Ethics: Implications for Africa
}

\begin{abstract}
This paper evaluates the potential benefits, drawbacks and ethical risks of cloud computing for African countries in the context of information communication technologies for development (ICT4D). The paper argues that the capability approach, incorporating development ethics, provides a useful framework for considering the ethics of cloud computing in Africa. Coupled with global and intercultural ethics perspectives, both provide a rich human-centred view of the technology's benefits, drawbacks and ethical risks. Focussing on the transformational benefits and features of cloud computing for small and medium enterprises (SMEs) and the health sector, the paper highlights potential ethical risks that are cause for concern. The paper concludes that while cloud computing has considerable potential for advancing development through the enhancement of capabilities, there remain huge challenges in its efficient, effective and ethical use. As a result, ethical risks related to equity, ownership, dependency, privacy, trust and security that reflect 'unfreedoms' and 'capability deprivations' may consequently have an impact on the technology's potential as an information communication technology for development.
\end{abstract}

Keywords: Cloud computing, Ethical Issues, Capability Approach, Intercultural Ethics, Africa.

\section{Introduction}

Cloud computing has transformed information and communication technologies (ICT) capabilities through new forms of hosting and delivering ICT services over the Internet. It presents African countries with the opportunity of leapfrogging infrastructural and provisioning problems by eliminating the costs of expensive hardware, enabling them to take advantage of hardware, software, data and platform services provided by service providers who own the technological infrastructure. Advancements in cloud computing offer cost-effective opportunities for handling data that enhances critical areas such as economic productivity, employment, natural disaster and resource management and public service delivery. However, socio-technical transitioning is often difficult to achieve due to various financial, political, economic, social and infrastructural factors.

Despite huge expenditure and investment in ICT in many African nations, financing and affordability remain a barrier to accessing the full benefits of cloud computing. More investment is required to finance the technological enablers and broader infrastructural capacities needed. Widely acknowledged capacity challenges include technological infrastructural issues (Estache, 2006; Foster 
\& Briceño-Garmendia, 2010; Kizza, 2009; Williams, Mayer \& Minges, 2011), which are exacerbated by limited broadband availability, computational capacities of mobile devices and power supplies (UNCTAD, 2013; ITU, 2012; ITU, 2015; Nkosi and Mekuria, 2010).

Other key challenges relate to the wider social and political contexts in which ICTs are introduced (Alzouma, 2005). Social challenges include illiteracy, lack of content and inequalities. The global political economy of ICTs has been characterised by processes of 'digital colonisation', involving ICT production and use initially in the global north, with culturally and economically hegemonic ideas being later 'exported' to African countries. Digital colonisation implies a dominant culture enforcing power and influence over minority cultures, and also takes the form of data sovereignty and disregard for digital data ownership and privacy.

Health and business represent two interesting areas in which cloud computing can bring considerable benefits, and this has been discussed in the literature (for example see Greengard, 2010 and Senanu, Ikhu-Omoregbe and Charles 2010). Greengard (2010) points out that cloud computing offers the potential for small and large business entrepreneurs in developing countries to overcome barriers occurring as a result of infrastructure difficulties. Kshetri (2010) argues that ecommerce and e-business are some of the major application areas of cloud computing in developing countries including Africa. With regards to the health sector, Nkosi and Mekuria (2010) argue that cloud computing can be useful in the provision of health monitoring. They argue that the technology can overcome problems associated with mobile health monitoring systems that, although being considered for use in health monitoring, fall short of computational and algorithm capacity. Therefore, cloud computing, they argue, can promote health service delivery to marginalised rural communities (p.633). Applied as an e-health solution, cloud computing overcomes challenges of data storage, difficulties in hospital-to-hospital collaboration, insecure information exchange and lack of accountability (Senanu et al, 2010). Cloud computing delivers a single application through the browser to thousands of clients or stakeholders using scalable multitenant architecture that helps minimize cost, manage healthcare resources effectively. Greengard (2010) argues that cloud computing also has the potential to level the playing field for businesses in developing countries by breaking down the barriers of entry, providing opportunities for creating new businesses and new business models as well as opening up new markets. Yet questions remain over potential value conflicts and the appropriateness of traditional notions of ethics for the new complexities that new ICT paradigms present in the health and business sectors. (Reidl, Wagner, \& Rauhala, n.d.; Stahl, Eden, Jirotka, \& Coeckelbergh, 2014).

The use of ICT to achieve health outcomes was reflected in the Millennium Development Goals (MDGs) on healthcare (p.1),) and remains a prominent feature of the Sustainable Development Goals (SDGs). Task 18 of the MDGs stipulates that 'In cooperation with the private sector, make available the benefits of new technologies, especially information and communications'. ICTs are also a powerful catalyst for realising SDG 3's aim to ensure healthy lives and wellbeing. While Cloud Computing can make important contributions to development (Buyya, Yeo, \& Venugopal, 2008), it also presents unique capability and ethical challenges.

We argue that the ethical dimensions of cloud computing in Africa have been overlooked (Dahiru, Bass, \& Allison, 2014) and governance systems remain weak (ITU, 2012). This paper therefore aims to explore the potential benefits, drawbacks and ethical risks of cloud computing from a capability and intercultural ethics perspective. Security risks, cybercrime and espionage are some of the risks that have been noted (Kshetri, 2012). Cloud computing poses potential ethical risks and drawbacks associated with privacy and data security. It raises many concerns including security, management and control, disaster recovery and business continuity, supplier management, regulations and 
legislations, and the lack of standards and guidelines (Carroll, Van Der Merwe, \& Kotze, 2011). Internationally, the legal implications of cloud computing have been highlighted (Székely, Dániel Szabó, \& Vissy, 2011). Privacy, trust and security concerns have been discussed elsewhere in the literature (Huang and Nicol, 2013; Habib, Hauke, Ries and Mühlhäuser, 2012; Marinos and Briscoe, 2009). There has been less attention, however, paid to these ethical dimensions and issues in the African context. The topic of information ethics in an African context represents a significant knowledge gap (Capurro, 2007b). Specifically, ethics in relation to ICTs for development is still underresearched (Mthoko \& Pade-Khene, 2013). Consequently, this paper considers the possible benefits, drawbacks and ethical risks of cloud computing for Africa as a potential technology for development.

The paper starts by discussing cloud computing in Africa, introducing the capability approach and highlighting its concern with development ethics. Intercultural ethics perspectives are then presented, and the methodology applied to the analysis is explained. The following section discusses the potential benefits of cloud computing followed by an assessment of the possible drawbacks of the technology. The implications of the ethical assessment for Africa are then set out, followed by the key conclusions.

\section{Cloud Computing and the African Context}

Historically, Africa has been beset with economic, technological and infrastructural challenges. Expenditure on science and technology during periods under structural adjustment programmes continued to be low (Enos, 2003), with little efforts directed towards technological learning (Wangwe \& Semboja, 2003). The adoption of cloud computing as an IT strategy in Africa is relatively new, and there is still the need to establish political and regulatory frameworks and appropriate training programmes as cloud computing is more popularised (ITU, 2012). Unlike in the global North, SMEs in Africa have been less concerned with challenges like security, privacy and data loss while showing great optimism for the opportunities cloud computing presents them (Dahiru et al., 2014). Conversely, health delivery organizations have been more reticent in using cloud computing technology in part due to their stringent confidentiality and security requirements (Samuel, Omisore, Ojokoh, \& Atajeromavwo, 2013).

Cloud computing may be regarded as 'a model for enabling ubiquitous, convenient, on-demand network access to a shared pool of configurable computing resources (for example networks, servers, storage, applications, and services) that can be rapidly provisioned and released with minimal management effort or service provider interaction' (Mell \& Grance, 2011, p. 2). In very general terms, it embodies hardware and software infrastructure that allows users with minimal software and hardware to store, retrieve and process data and information easily and efficiently to meet their technological needs (Dikaiakos, Katsaros, Mehra, Pallis, \& Vakali, 2009). In the African context, technology enablers for cloud computing that include the development of parallel programming paradigms and open source software, cloud access to high performance computing, Green IT, investment in low-cost access-cum-computing devices and hi-speed networks are in their infancy or non-existent (Dwivedi and Mustafee, 2010).

In a number of African countries there are evident cloud computing projects underway largely as a result of partnerships between international players and African economic operators (Weber, 2011; Nkosi \& Mekuria, 2010). However, as Nkosi and Mekuria (2010) point out, such projects are not without problems. For example the cloud computing for enhanced mobile health applications faces problems related to limited computational and power supply which has a direct impact on the secure delivery of health services. However, the human technical and financial resources available 
to African operators fall short of the requirements of the technology, including the implementation of training and institutional strengthening programmes (ITU, 2012). Despite these insufficient resources, network infrastructure, connectivity and use are increasing. Therefore although in many instances connectivity is far from sufficient for cloud computing, Abubakar (2016) argues that some companies and universities in Africa (such as the University of Pretoria) are using cloud computing for their businesses and research respectively. In addition, as UNCTAD's Information Economy Report (2013) has suggested, even in less developed countries where governments and business have not substantially adopted cloud computing, the use of cloud services by citizens is on the rise.

Companies such as Microsoft are making their expertise and programmes available to internet access providers such as MTN. A survey by ITU (2012) found that 50 percent of ICT operators in Africa have adopted cloud computing technology but with varying capabilities and attention to ethics. Literature on cloud computing particularly in Africa is scant (Kshetri, 2012), and information ethics a young academic field in Africa (Capurro, 2007a). There is still a need to better understand how ICTs intertwine with African ethical principles linked to communalism (Capurro, 2007a) and consider ethically the capabilities and needs of those whose interests are affected by innovations (Zheng \& Stahl, 2011) such as cloud computing.

\section{Capability}

Issues of capability in relation to technology have been addressed in the literature (Johnstone, 2007; Kleine, 2011; Smith, Spence, \& Rashid, 2011), often drawing on Sen's work (2001) on development ethics (Clark, 2002), which considers an individual's goals and wellbeing. The capability approach has grown in influence as an analytical lens for interpreting ICT4D research and practice (Bass, Nicholson, \& Subhramanian, 2013; Johnstone, 2007; Kleine, 2011; Smith et al., 2011) and the informational and networking capabilities of users (Gigler, 2011; Smith et al., 2011). As an ethical assessment applied to ICTs, the capability approach draws attention to values based on functionings (Johnstone, 2007) and its evaluative space comprises individuals and groups (Smith et al., 2011). The capability approach enables an analysis that expands the definition of ICT4D goals, from an emphasis on economic goals conflated with material prosperity to include a focus on individual fulfilment (Bass et al., 2013). It identifies capability, functionings, conversion factors, freedoms and values applied to resources (Kleine, 2011; Sen, 2001). The capability approach views cloud computing as a resource, that expands human capabilities and enables achievement of valued goals, rather than viewing access to cloud computing as intrinsically good in itself. The capability approach demonstrates that technologies are not neutral, but rather imbued with values that may not be complimentary with those of users nor be of benefit to them.

In broader technology studies, the term capability tends to refer to individuals' ability to use technologies, or attain functionings in relation to technology use. Functionings refer to actual achievements that individuals or groups have reason to value (Sen, 2001). Functionings in this context would include outcomes achieved through the availability of cloud computing, such as improvements in technological, infrastructural and organisational capacities to achieve business and health goals. It would also include intermediate achievements such as increased information, enhanced connectedness in the service of health and business goals. Functionings also comprise proximate business and health benefits that are valued and meet national ICT4D goals of healthy individuals and thriving businesses enabled by expanded connectivity and increased information flows mediated by cloud computing. Conversion factors represent what and how functionings can be attained from resources by virtue of personal circumstances and external conditions. Robeyns (2005) articulates these as personal (for example, skills and intelligence), social (including public 
policies, social norms, power relations) and environmental (for instance built and physical environment) influences that enable the conversion of resources into functionings. In the context of cloud computing this would involve ICT skills, policies, processes and infrastructures. In Sen's approach, capability refers to the freedom to achieve goals, to 'be and do' (2009). Freedoms might be economic, social and political in nature and also include transparency guarantees and protective security. Sen (2001) argues that expansion of freedoms should be the primary end and principle means of development and that the removal of 'unfreedoms' is constitutive of development. Typically, national ICT policies and goals in Africa aim to expand human capabilities through access to ICT services to facilitate development.

Although critics have pointed out its limitations (see discussion by Alkire (2008) and Clark (2005)), we suggest that the capability approach is useful for evaluating the benefits and drawbacks of cloud computing from a computer ethics perspective. Computer ethics is concerned with 'the protection and advancement of central human values such as life, health, security, happiness, freedom, knowledge, resources, power, and opportunity' (Bynum, 2008, p 36). As such, it shares with the capability approach a human-centred focus, value-based agenda and a concern for the role of public policy and investments in meeting human needs. A focus on capabilities with regard to computer ethics considers the ability to use cloud computing free from ethical infringements, and to attain functionings through its use without ethical interferences. Notions of capability, as freedom to protect and advance human values through cloud computing application and use are implicit in both approaches.

The capability approach allows an analysis of the ethical dimensions of cloud computing by providing an evaluative space for considering ethical values and principles such as equity, well -being and responsibility. The capability approach spotlights value judgements in the identification and prioritisation of ICT resources, capabilities, choices and alternative courses of action related to the adoption and use of cloud computing. Such an analysis questions which capabilities are expanded for whom, how and for how long (Alkire, 2008). The capability approach allows a theoretical framing for research exploring how ICT impacts on human capabilities (Coeckelbergh, 2011). As an evaluative framework, the capability approach enables the assessment of benefits and drawbacks of different courses of action or state of affairs. Benefits within an ethical assessment of cloud computing would be treated as capability enhancements and drawbacks as deprivations/contractions of capabilities. Contractions of freedoms and capabilities might include the invasion of privacy, misuse of personal data and disputed ownership of data (Johnstone, 2007).

Cloud computing may raise different culturally nuanced and contextualised ethical issues in Africa when compared to the global north. It is important to recognise these variations in order to gain a comprehensive understanding of the ethical concerns of cloud computing. We argue that the value of the capability approach lies in the way it enables us to ask a different set of questions about cloud computing, such as which capabilities are realised and which 'unfreedoms' might arise. Cloud computing presents numerous possibilities for Africa insofar as the capabilities it delivers relating to overcoming hardware and software challenges, as well as accessibility to easy and faster networks. However, the benefits of cloud computing are largely optimistic, unspecified and under-theorized. Cloud computing is regarded as a capability good and a functioning broadly equated with equity and freedoms, yet these are questionable particularly when we look closely at wellbeing, security and responsibility concerns. This paper therefore considers the ethical imperatives and dimensions of the cloud computing phenomena.

In considering the potential of cloud computing one must consider its features (which include shared resources, electronic collaboration, data storage, efficiency and cost-savings) to establish to what 
extent cloud computing impacts on human development, agency, wellbeing, freedom, security and responsibility. A focus on these facets of the capability approach is important in several ways. First, a human development lens shifts the focus to communities' needs and goals, and helps us better understand whether users are able to achieve development functionings through cloud computing. Second, the concept of agency highlights the extent to which cloud computing users are able to 'be and do', free from ethical challenges. Third, wellbeing spotlights the extent to which cloud computing is instrumental in achieving indicators of wellbeing, such as freedom from privacy and security contraventions. Fourth, freedom draws our attention to whether cloud computing advances or curtails the freedoms of users and the nature of these freedoms. Fifth, security focuses our minds on the kinds of security and insecurity cloud computing presents to users. Sixth, responsibility interrogates the extent to which data protection safeguards and other governance structures are in place and adhered to, as well as how stakeholders ensure greater responsibility and accountability when it comes to ethical concerns arising from the use of cloud computing.

As responsibility lies with a range of actors worldwide, an intercultural ethics approach (explained in the next section) to addressing global ethical challenges around cloud computing-related capability deficits and unfreedoms is necessary. Combined with intercultural ethics, the capability approach provides a useful analytical lens for investigating the ethical dimensions of cloud computing, through a two-pronged approach. The capability approach illuminates capabilities, unfreedoms and ethical values related to cloud computing. Intercultural ethics focuses on similarities and differences across cultures (Capurro, 2008) and the need for shared norms, values and practices across cultures and for shared global ethical solutions.

\section{Intercultural Ethics}

We incorporate intercultural ethics as an additional analytical framework as it relates to culturally contextualised ethical dimensions of cloud computing, shared global concerns and the need for global responses and moral considerations. Ethics differ according to culture, and a cross-cultural awareness of ethics provides a rich learning opportunity (Evanoff, 2006). This helps to 'gain a wider and more highly differentiated view of the world which can nonetheless be integrated into a wider and more comprehensive worldview' (p. 422). We define intercultural ethics as trying to reach a shared understanding of ethics across cultures through a dialogical process while recognising some degrees of divergence. Arriving at some shared understanding of ethics in the context of cloud computing is important, considering that the owners of cloud computing platforms are largely based in wealthier countries. In the current global climate, asymmetric power relations between developed and developing countries and increasing efforts to access citizens' private data mean that poorer countries' ability to influence privacy policies, for example, is constrained. Bell and Pavitt (1997) have illustrated how asymmetric power relations can result in their discussion of contrasts between developed and developing countries when it comes to technological accumulation and industrial growth

As a global phenomenon, cloud computing requires a global understanding of the ethical challenges it poses. Capurro (2008) calls for intercultural information ethics that addresses similarities as well as differences between different cultures. This can be particularly true for cloud computing in an African context. Cloud computing provision is strongly influenced by hegemonic Western values concerning the use and adoption of technology from the design phases, and technology policy. Thus far, little attention has been paid to ethical concerns of African users specifically. Many African cultures typically emphasize values of communalism and a recognition the humanity of others, 
which is in contrast with the way in which ICT use has been framed in Western cultures as based on the assumption of individualism (Capurro, 2007a).

The concept of ethics as a reflection on morality is widely accepted (Capurro, 2007a), with ethics described as "a rational examination into people's moral beliefs and behaviour" (Quinn, 2006 p. 55). This examination of the moral standpoints, values, principles and practices that are ascribed to everyday life will inevitably include cloud computing, which is increasingly becoming an intrinsic part of our everyday lives. As computers are used for information processing, information ethics principles can be applied to the production, storage, access and dissemination of information (Ess, 2007). Therefore, it is important to critically evaluate ethical implications in relation to different technological phases from development to implementation stages right up to the time they are appropriated for use and the extent to which use upholds moral values and ethical norms, principles and obligations. Cloud computing deployment, adoption and use need to take into account any potential ethical challenges that may arise.

There is some discussion in the literature exploring a global information and computing ethics framework that incorporates shared norms and values, practices and traditions of different cultures (Capurro 2007b, 2007b, 2008 and Ess 2007). Development of such a framework would demand assessments of ethics that are flexible in their application and lend themselves to ethical evaluations across cultures. Legal rules, institutional solutions and national policies (Székely et al, 2011; Sen, 2001) are one way in which ethical norms, principles and obligations are expressed and necessitate seeking out commonalities and pragmatic agreements based on shared interests (Ess, 2007) for the global good. Whilst variances in cultural context and local need are important considerations, identifying ethical issues at an earlier stage in the development process is advantageous. Improvements may then be considered during policy formulation and implementation. Concerns to do with policy formulation and implementation that go beyond use to cover issues of access, capability, privacy and security can be mitigated for cloud computing in African countries through regulatory instruments and risk management.

\section{Methodology}

Using qualitative data from interviews conducted with African and international Information Systems (IS) professionals and grounded in the literature on cloud computing, the capability approach and intercultural ethics, this paper uses an interpretive approach (Walsham, 2006) to explore the possible benefits, drawbacks and ethical risks of cloud computing for Africa as a potential technology for development. Although there is a growing body of literature on cloud computing and ethical issues (for example Wakunuma \& Stahl, 2014; Tatten, 2012; Zissis \& Lekkas, 2010; Timmermans et. al, 2010; Stahl et al, 2010), there is a lack of research looking at the potential for cloud computing to contribute to positive development outcomes in Africa in the context of ethical concerns. Consequently this paper is based on two research activities, firstly investigating future and emerging technologies and associated ethical issues, and secondly looking at the identification and governance of emerging ethical issues in the field of information systems management.

Eleven emerging technologies and their associated ethical concerns were identified, including cloud computing in the first research activity. Although there was an international cross-cutting textual analysis of policy and scientific visions of future technologies, the research validation processes (including focus group discussions a survey of ICT research projects and expert 
workshops), appeared to be Eurocentric rather than international in its focus. Although this previous work provided an overview of the issues, it lacked a more in depth discussion of the possible benefits and drawbacks of cloud computing as a technology for development from an African point of view. Follow-up research was more focussed, with a total of 26 in-depth interviews along with survey questionnaires conducted with Information Systems (IS) professionals on their experiences and perceptions of cloud computing. The participating IS professionals came from the following countries: Australia (1), Canada (1), China (1), Finland (1), Ghana (1), Hungary (2), India (1), Malaysia (1), Malta (1), Nigeria (1), Trinidad (1), United Kingdom (13) and Zambia (1).

\subsection{Data Collection and Analytical Approach}

The follow up research project involved an initial survey questionnaire sent out to IS professionals covering generic questions on demographic information such as gender, age, location and education. Survey participants were all male and situated in the locations identified above, which included 3 participants from African countries. The respondents all held management positions and had a university degree in a related IS field. Their ages varied between $31-60$ years. After the survey, in-depth interviews were conducted in order to gain a more detailed understanding of participants' experiences and perceptions of emerging technologies and related ethical issues (see Appendix A).

The low number of participants from African nations in this study is indicative of lower numbers of IS professionals in these countries and their relative lack of experience using cloud computing within the region, and is demonstrated by participants' framing their responses in terms of perceived benefits and risks rather than basing their responses on their direct experience. However, we maintain that an analysis of African participants' responses and experiences in other countries serves as a springboard for further discussion around the possible benefits, drawbacks and risks of cloud computing in relation to Africa. The participants' insights suggest that applying capability and intercultural ethics approaches to cloud computing is a valuable way forward.

Drawing on the idea of intercultural or global ethics as a methodological device (Capurro 2008, Ess 2007), the data was analysed in terms of similarities and differences across developed and developing regions (Capurro, 2008) then shared norms, values and practices across cultures (Ess, 2007). Findings were then categorised under potential benefits and ethical issues, which are presented in the next section in terms of their implications for African countries. Issues raised in the initial analysis might be regarded as Eurocentric. However, in exploring ethical issues in relation to Africa, many commonalities and synergies were identified. Developed and developing countries are vastly different environments, for example, developed countries may be more advanced in terms of technological skills, development and knowledge (which has implications for the adoption, access, use and affordability of technology), and experience different ethical issues than African countries. We argue that there has historically been a flow of knowledge from north to south in most if not all areas related to the technology. However, even as the same technology may be adopted in different country contexts, its practical application may vary due to social, economic, political and cultural differences which may include discrepancies in access, affordability, knowledge, relevance and need. With this in mind, the following section outlines some of the potential benefits and shared global ethical issues of cloud computing that are relevant in the African context.

To enrich the discussion on the implications of cloud computing for Africa, in this section we also consider the data collected from IS professionals in developed countries in order to look for possible contextualised differences in the way problems are experienced and addressed in developed and 
developing countries. We focus on the benefits, drawbacks and ethical issues raised that apply to both developing and developed countries, emphasising the context, realities and implications for African countries. Common global themes include capability expansions, human, technological and organisational capabilities and shared informational and knowledge capabilities. Shared global ethical concerns include security, privacy, trust and data control and ownership, equitable access, inclusivity and vulnerability to hacking and technology dependency. We draw on these commonalities precisely because there has been little analysis of these in issues in the context of Africa. Concerns articulated by respondents in developed countries include concerns about loss of jobs and espionage, while respondents in developing countries were concerned about cost, surveillance and censorship. A focus on the similarities rather than the differences in responses reflects our argument for a global response and an intercultural approach to ethical issues experienced by both African and non-African countries, whilst acknowledging the additional constraints faced by African countries.

\section{Potential and Benefits}

\subsection{Leveraging the Benefits of Cloud Computing for Capability Expansion}

In general, participants acknowledged that cloud computing has the potential to transform organisational resourcing and use of ICT, contributing to advancing international development goals by shaping the way ICT infrastructure is designed, purchased and deployed, and advancing the informational and networking capabilities of users (Gigler, 2011; Smith et al., 2011). Innovators no longer face large capital outlays in hardware to deploy services or the human expense to operate them. Cloud computing has the potential to prevent the wasteful overprovisioning of services that do not meet needs, and the under-provisioning of unexpectedly successful services. The costeffectiveness of cloud computing is a key issue, as highlighted by this respondent:

Well, cost is a major consideration for us these days. If we do not have our own infrastructure, we are buying our own fire-wires, buying our own data centres, you know. Now everybody is looking at buying software as a service. You subscribe to services and only pay for actual usage as opposed to having your own infrastructure and applications and have people managing all this. That's a radical departure from the current practice. A lot of people are beginning to embrace it because of the obvious alternatives in terms of managing cost.

Large ICT processing tasks achieve results as quickly as their programs can scale an elasticity of resources, without paying a premium. The exciting possibility of optimising resource use is reflected by a respondent explaining 'that you use only those resources you need at the moment is fascinating'. This particular participant appreciated that they did not need to spend resources on hardware or software if there was no need to, which demonstrates an expansion of choice, freedom and capabilities (Sen, 2001). All they needed was to utilise the services of a cloud provider as and when necessary. This sentiment was also expressed by an African IS professional who had the following to add:

I look at [cloud computing] and see the possibilities in the near future. We may have to forgo some of our computers and also send our data for processing on the Internet. I am looking 
at [cloud computing] so that we will not waste funds you know, because of technology we will be able to guard against it.

This quote illustrates how cloud computing can potentially increase choices, freedoms, capabilities and associated functionings pertaining to health and business. Africa's health delivery systems have faced technological challenges related to data storage, securing patient medical records and accessing quality healthcare services, accurate diagnosis and therapy (Samuel et al, 2013). Cloud computing has the potential to transform public services and expand freedoms in the health sector by providing a business model for efficient delivery of health services to support health and wellbeing goals. It also has the potential to relieve mobile devices from executing heavier multimedia and security algorithms in delivering mobile health services (Nkosi and Mecuria, 2010). This contributes to expanding healthcare delivery organisations' capabilities and achievements in relation to wider health and social care interventions and health improvements.

Africa's existing technological infrastructure has for a long time been singled out as one of the major barriers to harnessing technology for economic growth (ITU, 2007). Cloud computing allows smaller businesses access to more efficient and optimising technological infrastructures. This potentially expands their capabilities in relation to market expansion, geographically and materially. Smith et. al. (2011) have demonstrated how ICT expands freedoms in relation to economic networks, connecting users with financial institutions, expanding market boundaries and improving supply chains. Interviewees felt that cloud computing would be beneficial for SMEs because it would 'bring up and showcase SMEs ... so that their products and services can be bought up by anyone over the Internet'. Added to this, it was thought that such companies could 'leverage the benefits for collective benefits to our economy'. As observed, technological advancements allow for ecollaborations which are advantageous for facilitating and expanding both local and international trade notwithstanding the promotion of trade as well (Goonatilake, Herath, Herath, \& Tyska, 2009). This means that African businesses can take advantage of this possibility to build new business relationships as well as to acquire and share knowledge in their area of business. Evidence of capability expansion through cloud computing evidenced in developed countries illustrates the enormous potential of cloud computing. African IS professionals also acknowledged this potential as cloud computing becomes more widespread in African countries. This may be particularly pertinent in both the health and business sectors. In health, cloud computing would be especially helpful in the easy access and exchange of patient data, while businesses would be able to leverage the cost-effectiveness of cloud platforms and avoid the costs of owning IT infrastructure.

\subsection{Transforming Technological and Organisational Capabilities}

Initial research as part of this study, explained in the methodology section, also found that cloud computing has the potential to expand human, technological and organisational capabilities to support international development efforts (ETICA project, 2009-2012), that enable functionings (Sen, 2001) related to process, operations, scaling and sharing. Research participants identified the opportunity for resource/storage virtualization where resources and services are delivered to users and/or organizations via Internet from resource clouds where almost all information and tools are preserved. Resources can be added or removed based on the need with services provided by "pay per use pricing" model. Data could be stored in multiple physical locations across many servers around the world with potential interconnections of multiple services across the cloud. At different levels, the functionality of different providers is connected to provide a specific service to an enduser. Economies of scale were another cloud computing capability enhancement noted. Because 
organisations and users of cloud computing services do not necessarily need their own hardware, software and platforms, the technology can potentially offer an economy of scale.

Interviewees also highlighted the benefit of shared use facilitated by cloud computing. As the technology can be used simultaneously by a variety of people, this means that the service is flexible enough to meet different users' needs. The ability to be able to share such a service is advantageous for the efficient usage of limited resources. Shared use also allows for the monitoring of work by different groups in order to maximise productivity. Where individuals or organizations may previously not know where information or services are coming from or stored, cloud computing fosters an element of belonging to a community of practice where users may feel that they are not working in isolation but as a group with common goals.

Capabilities linked to accessibility, ease of usage, fast information sharing, delivery and control were also noted by interviewees. Ease of use of cloud services is emphasized in many scenarios, one of which is illustrated by an interviewee's comment about organisational capabilities and flexibility:

Technology changes very rapidly. If you maintain your own infrastructure, the challenge you face is that you must keep tabs on new developments you must ensure that your people are abreast of current technologies. And you have to have the required skills in house to manage and rectify these things. And there is only so much you can do with your in-house staff. The rate of attrition in this industry is quite high, so when you lose your key staff, what happens? How easy is it to replace them? But if you are buying service from a legitimate service provider, the service provider will then be dealing with each challenge and you can focus on your core. If you are a financial institution you are not an IT organisation so you want to be able to focus more on your business, rather than purely managing infrastructure.

The cloud computing service experience is assumed to be smooth and quick with very fast and optimized connections. As users to not have to install anything on their own computer, it is assumed that they will always have the most suitable version of the software and service and that the incompatibility with products and services will not be a problem. On the other hand if the system or service updates automatically then service could also appear as totally different every time one uses it.

We suggest that there are particular areas where cloud computing might be applied in Africa and enhance functionings. For example, nomadic business organisation (where businesses and other organisations are able to operate fluidly by being able to access information from anywhere and at any place) would stand to benefit from cloud computing. Cloud-delivered environments will also benefit organisations where the shared usage of resources and information is important, particularly for organisations work in partnership with each other but are based in different locations.

\subsection{Shared Informational and Knowledge Capabilities}

Gigler (2011) has illustrated the importance of understanding informational capabilities in terms of ability to use ICT in an effective manner to find, share and produce information, and generate knowledge. Research participants in this study pointed out the increased prospects for knowledge sharing that cloud computing provides in terms of information flows and sharing. As one participant suggested: 
Cloud computing is important particularly when we talk about pools of shared information, where information is hosted, and ERM management of the infrastructure and the flow of that information.

As cloud computing allows for shared resources, it can be said that such a capability might result in a cut in hardware as well as software costs. For Africa especially, "sharing" is a trait recognised at different levels and in different situations. As Heeks (2009) intimates that sharing fits in with the Southern pattern of shared ICT access 'where - for example in the local cybercafé - users cannot guarantee to be using the same client device every time (2009, p. 7). Cybercafés have for a long time allowed users who have not been able to afford internet access at home to be able to have access through such platforms. Additionally, the widespread use of mobile phones in both urban and rural areas across Africa allows those who cannot afford their own phones to share with neighbours, relatives or friends in their communities. Cloud computing architecture is therefore a new focal point that allows for shared access.

As highlighted previously, cloud computing enables easy access to data storage as well as delivery. This allows clients to be able to access services much more easily and quickly at a potentially lower cost. In addition, because this is a service provided by other organisations, clients will not necessarily have to worry about IT maintenance and associated updates. This provides opportunities to expand the technological and human capabilities of many African countries to support broader development aims, but also raises some concerns that need addressing before this potential can be realised. With this in mind, the following section will look at some of the ethical considerations relating to cloud computing, with a particular focus on the implications for Africa.

\section{Drawbacks}

Results from the research suggest that although interviewees perceived cloud computing as beneficial especially with regard to saving and optimizing the use of resources, they also voiced concerns relating to information ownership, trust, privacy, equality of access, technology dependency and addiction, security and privacy that represent forms of 'unfreedoms' and capability contractions (Sen, 2001; Johnstone, 2007).

\subsection{Security, Privacy, Trust and Data Control and Ownership}

Both developing and developed countries have similar concerns about the ethics of cloud computing in relation to security, loss of privacy and control of data. These issues are of global significance as cloud computing technology is fast filtering across borders. The desire not to worry about expensive infrastructure and the need for easy and fast information sharing are attractive in African countries whose IT development has for the most part been lagging behind due to infrastructure difficulties. However, as interviewees particularly from the developing countries pointed out, cloud computing is a concern due to the potential loss of control of one's data as the data is stored and managed by service providers in developed countries. This has implications for not only security of data but also privacy.

The fact that information is stored in "clouds" was a central issue for interviewees, particularly when it came to the question of who owns the information and how that information can be used. Participants felt that if all the information was kept at one place by the service providers, the service providers would have control over it. The question of trust between service providers and customers 
was consequently a key theme in the interviews. The fact that third parties would hold their clients' data was a concern for interviewees. They indicated that although they had no doubt there would be assurances of trust, it would be difficult to see how this would be upheld, especially as there could be opportunities where some elements might unwittingly mismanage data thereby causing security concerns as well. The question therefore is how service providers would manage the risks that may result without compromising client data. Participants also felt that where trust could be compromised, threats to privacy would follow. Questions of how service providers would address privacy concerns and adequate policy responses were some of the concerns raised, as illustrated below:

I am aware we've moved a long way away from when I started in computing during the late 60 's. The issue [now] is understanding for instance, where your data actually is - on a computer system or computer network. I think we've long since gone away from it. Part of my job is computer security, trying to understand exactly where there are copies of data, and where they're cached and all the rest of it. With modern technology I mean you can almost forget it. I mean, if you allow your data onto the Internet unencrypted, you have totally lost control... What you are doing is moving the envelope of trust to whoever runs the cloud and the communications between you and the cloud.

Well, it depends on the sensitivity of the data. If you are trying to ensure confidentiality of the data, then how much do you trust the computer operators, whoever is running the cloud? It doesn't matter if it is a well-known company, you know, Microsoft or Google or somebody or whether it's a small one. How do you gain that much trust? I mean bearing in mind again, that you aren't quite sure whether your data is being stored, shall we say in Britain or [somewhere else] because it's cheaper.

The interview extracts above illustrates the anxiety of this IS professional around the loss of control and power associated with another party managing the cloud services used. This particular interviewee (above) expressed the belief that service providers wish to store and manage data as cheaply as possible, with the user's data security a secondary priority. In her work, Mansell (2010) discusses how unequal power relations can occur in technology and innovation. She argues that systems of innovation processes encompass inequality and power dynamics that influence the distribution of resources and the new spaces of opportunity and insurgency available to any given actor (p. 17). It is therefore unsurprising that the issue of trust and concern for loss of control is identified as a concern in cloud computing:

Security, security is key. If you are a financial institution and you are trusting your most important asset - data or information - to a trusted third party, you must ensure that this trusted third party is in a position to guarantee the integrity and confidentiality of your information. More importantly you have to ensure that this trusted third party is in a position to provide you with a service all of the time, and reliability is key. If you have your infrastructure, and you have your own people managing your infrastructure, you can be rest assured that in the event of any mishap, you can bring to bear a sense of urgency, seeing as you are insiders. You are in control and you manage them. But if it is a service provider, to what extent will [they be] in a position to provide you with the requisite level of service? And 
in the event of any breach [of security], what recourse is there for you? What kind of arbitration would be required? The legal framework, do we have appropriate laws to guide such things?

When it comes to solutions, the general consensus of the participants was the need for robust regulations and workable policies that would help in overcoming some or all of the ethical issues pointed out. In addition, interviewees suggested that users and service providers needed to come up with proper auditing and management processes that the parties involved would adhere to as a way of having certain degrees of assurance and trust should anything go wrong.

Other interviewees showed rather more optimism about the potential of shared ownership:

What I am hoping will happen here is that the organisations in the industry will perhaps come together and begin to push the idea of shared services. For example, imagine what would happen if all the major banks decide to collaborate and pool resources to create a shared infrastructure, rather than every organisation building their own. I think the air travel industry is a model that is already working well. They already have shared infrastructure. No single airline has airports or control towers in every major city. These are all shared infrastructure. When you are travelling you really don't care who takes you. You go to an agent and you book your itinerary and they take you to your destination. You really don't care who owns what infrastructure along the line.

The ICT policies of many African countries do not address aspects of cloud computing or for that matter ethical issues. This is however not surprising, particularly when we consider that cloud computing is a relatively new technology in the region. ICT policies generally frame such technologies in a neutral sense, neglecting to sufficiently address ethics, issues of security, trust and affordability (Britz et al, 2013). Such omissions might make it difficult for stakeholders involved in the provision of cloud computing to develop robust regulations and workable solutions as well as trust should anything go wrong. Additionally, some government policies, such as the increasing calls to register prepaid mobile SIM cards (GSMA, 2013) may be seen as government interference in the freedoms of users to be able to protect their information from the state. Obligations to register mobile SIM cards indicate the possibility that governments may also produce similar legislation relating to data stored and shared in the cloud, which brings up issues of control and ownership not only by cloud computing providers but also by state machinery.

\subsection{Equality in Access and Inclusivity}

A key difference between the research participants from developed and developing countries was the issue of costs and loss of jobs. Developing countries were more concerned about the affordability of new technologies as historically they have tended to be more expensive for developing countries at the point of introduction. Participants commented that it is not desirable for users to be offered access to the services without fully understanding the 'behind the scenes' operations. Some interviewees suggested that users should be able to understand the system and be able to use the service according to their own skills. Without this, they felt such a technology would result in miscommunications and inequality of access to services on offer, not only within the IT-literate population, but also between laypersons and service providers. Furthermore, although they acknowledged the potential benefits of cloud computing, some research participants were concerned about a general lack of computer skills as well as accessibility and how this might affect the use of cloud computing in Africa: 
We have so many people who [are not] able to use the computer still you know. Cloud computing is coming and we have to make sure we [understand] this area and that computers are beneficial to us.

This suggests that the effective adoption of cloud computing resources requires attention to human capital investments. Robeyns (2005) argues that skills are important influences that enable the conversion of resources into functionings. Capability deprivations such as the lack of computing skills and access to computing technologies raise questions about whose capabilities are expanded and who benefits. Additional courses of action such as up-skilling and basic technology resourcing that need to take place in order for more equitable, sustainable, socially just and effective use of cloud computing in Africa.

\subsection{Vulnerability to Hacking and Technology Dependency}

Vulnerability to hacking and technology dependency were other drawbacks cited by research participants. The issue of hacking the cloud is a global concern of heightened significance to African countries on account of low technological skills. As Subashini \& Kavitha (2011) reveal, web applications such as the cloud introduce security risks such as hacking which at the time of their study accounted for $59 \%$ of the breaches. Recent media reports on high profile cases involving the alleged hacking of iCloud accounts have raised serious concerns in developing countries, particularly when it comes to medical and business-sensitive information that may be stored in the cloud. Additionally, the stories counter any enhanced security cloud models as suggested by Samuel et al (2013) who while acknowledging the difficulties of healthcare deliveries in developing countries more generally, also principally acknowledge the security risks posed by cloud computing in securing medical data suggest a Data Security and User Authentication Engine to prevent authorised entry to patient medical records. However, with determined hackers, such a model may not be very reassuring especially when we consider how such a model can be sustained as well as the low technological skills that are prevalent in Africa.

Research participants also raised the question of what happens when users encounter problems when they have become too dependent on the technology especially in relation to interoperability of devices and systems. In particular the issue of reliability of virtualised services was raised. Participants suggested that there was a danger of users becoming addicted to technologies like cloud computing which would to a certain extent leave them vulnerable to privacy problems for if they became too complacent and reliant on others for service provision.

We have discussed the possible benefits and risks associated with the uptake and appropriation of cloud computing in the African context, particularly with regards to positive development outcomes. We now go on to consider the ethical implications for Africa.

\section{Cloud Computing and its Ethical Implications for Africa}

In critically analysing the ethical issues posed by cloud computing from an intercultural and global ethics lens, it is clear there are commonalities with respect to shared concerns as well as differences 
in perspective based on differing contextual realities and capabilities. Ess's (2007) call to reconcile shared norms with individual and cultural differences is significant for African countries. If information ethics is to be global and achieve normative legitimacy it must address differences but also provide a shared response to central ethics problems (Ess, 2007). Cloud computing opens up new spaces for action and experiences and for enlarging capabilities, but there are significant risks and challenges. Here we focus on five key global ethical considerations of importance to African countries that highlight the complexity of pluralist ethical frameworks.

\subsection{Ownership}

While it has been suggested that African countries are less concerned about security, privacy and data loss (Dahiru et al., 2014), ownership of information is a global concern for cloud computing users regardless of geographical location. Although data and information ought to be the preserve of the client, the fact that service providers are responsible for it (and by implication have control over it) raises concerns of autonomy and freedom to one's information. In the context of Africa, it particularly raises claims of digital colonialism. The identity and location of service providers is of key interest to African businesses. Most service providers of cloud computing are located in developed countries and are large international corporations such as Microsoft, Google, Facebook or WhatsApp. This may hinder the development of local ICT developers who may be reliant on more developed countries to provide the technological expertise. Therefore the issue will go beyond concern of ownership to concerns of reliance, particularly as providers may not necessarily be local providers but corporate entities from developed nations. Such reliance may also lead to a loss of closer, traditional business ties based on face-to-face trust-building interactions.

\subsection{Trust and Privacy}

Trust, Molony (2006) has argued, is an important aspect of ICT usage within African enterprises, since traditionally doing business through face-to-face social interaction has been key. The fact that cloud computing services may be provided without this face-to-face contact may thus prove problematic for some enterprises. In this sense, trust an issue associated not only with control of client data and security, but also relates to establishing social relationships. Potential new forms of business relationships afforded by cloud computing such as online business interactions pose challenges for maintaining trust where there is physical separation, but as Mukherjee and Nath (2007) argue, successful relationships can be established and maintained through antecedents of trust (privacy, security, shared values) which influence relationship commitment.

Dialogue around concepts of trust highlights the question of whether new conceptualisations of privacy are required in African countries. Indeed, Ess (2007) suggests there might be irreducible differences related to identity and culture as compared to those in developed countries. Conceptions between private and public no longer work in the context of cloud computing technologies that facilitate ubiquitous systems (Matzner, 2014). Their pervasiveness makes it more difficult to establish which information will be processed by whom. Context-based privacy norms (Moor, 1997) are difficult to maintain where the meanings of privacy are continuously in flux (Matzner, 2014) in relation to cloud computing impacts on information processing, storing and disseminating. Tensions between individual rights and national security, owing to political developments and the revelation of surveillance by government agencies (Stahl et al., 2014) are 
likely to be a significant issue impacting on trust too. Ethically assessing security practices and principles highlights problematic power relationships.

\subsection{Equality and Digital Divide}

In the African context, a key determinant of whether equality can be achieved is affordability, that is, whether businesses are able to afford the services that come with cloud computing. Any gap between those who can afford access and those who cannot will likely exacerbate and not reduce the digital divide within African businesses and those outside of Africa as well.

It is also questionable whether cloud computing fosters new inequalities in terms of information, knowledge and skills relating to the use of data, technology and services, and also privacy. The capability approach highlights the significance of how technologies are used and the skills available to use ICT effectively (Coeckelbergh, 2011; Johnstone, 2007), so that the digital gap is less about access and more about securing positive outcomes, including the right to privacy and to development.

Ethics is important when considering ICT as a conduit of development in African countries, as ICT has a greater impact than other technologies (Mthoko \& Pade-Khene, 2013). ICTs are an ever present, dynamic and frequently changing facet of daily lives spanning business, education, leisure, health care, politics and many more areas, and are likely to continue to play a significant role in society. Development ethical frameworks that address issues of inequality and exclusion connected to cloud computing services are going to require some attention to advance the capabilities and functionings, as outlined in the capability approach (Nussbaum, 2001; Sen, 2001, 2009). Cloud computing as a tool for accessing information and services can inadvertently propagate inequality and exclusion.

\subsection{Technological Infrastructure Issues}

Major uptake of cloud computing might not be a reality for many small scale African businesses due to limited internet and broadband access. Therefore, if accessing cloud computing calls for accessing of services via the Internet, the Internet infrastructure would have to be improved vastly in order to make a major impact for African businesses. If this is not possible, then the issue of inequality and consequently the digital divide will continue to expand not only within and between African businesses but also between Africa and the developed world. Collaboration and shared resources might be affected as a result. In their white paper, McCalla et al (2009) confirm these concerns by indicating that without robust broadband access or accelerated deployment of affordable broadband, small and medium-sized businesses might not improve their productivity. This is because in an environment of wider ICT growth, such business may fail to connect with their customers; farmers to their markets; students to quality education; villagers to modern healthcare; and communities to each other to address a vast array of interests (McCalla et al., 2009, p. 1). In a similar vein, Williams (2010) argues that although sub-Saharan Africa, for instance, realises that ICT is necessary for long term economic growth, limited broadband connectivity due to high prices and unavailability are a hindrance to such growth and economic development. This, Williams (2010) argues, is also assuming that once there is availability and cost, there will be uptake of the 
technology, policy implementation permitting. In essence, Williams is also arguing for policy frameworks and standards that will ensure that the deployment of technologies like cloud computing become a reality. Lastly, Armbrust et. al. (2010) have pointed out that aside from technical failures, there is the possibility that cloud computing providers will suffer non-technical challenges such as going out of business or being caught up in regulatory quagmires that might impact the business. This may have a negative impact on clients reliant on the providers.

\section{Conclusion}

This paper has outlined the potential benefits, drawbacks and ethical issues associated with cloud computing, with particular focus on Africa. As an evolving paradigm for managing and delivering services over the Internet, cloud computing has the potential to enhance capabilities in relation to ICT4D. However, there are some concerns relating to development and computer ethics that policymakers need to address, and which represent unfreedoms and capability deprivations. Policymakers have a responsibility to minimise the negative impact of cloud computing on the advancement of human capabilities. This is a dynamic process, given that ethical frameworks relating to emerging technologies are in continual flux, responding to new ethical and governance challenges. A better understanding of the contextualised ethical challenges related to cloud computing and subsequent governance challenges may help policymakers develop solutions through building global partnerships focused on these challenges. A global information and computing ethics grounded in intercultural information ethics (Capurro 2008) that addresses shared norms and values, practices and traditions of different cultures (Ess, 2007) may support the enhancement of capabilities and mitigation of unfreedoms to support international development goals in Africa.

Cloud computing facilitates international development goals linked to human development, agency, well-being, organisational capabilities as well as infrastructural and provisioning freedoms but has privacy, trust security and accountability risks. By enabling businesses' access to technology, cloud computing serves to achieve human development goals of providing goods and services as well as wealth creation. y By removing access and sharing barriers between clinicians, thus providing more efficient health delivery, cloud computing contributes to human development goals linked to health. However, the level of entry barriers for businesses and health providers can come at a cost, which may include privacy, trust and security unfreedoms.

With cloud computing, organisations in Africa are presented with the capability to be able to choose how they go about doing business, who they do business with and how they exchange information without worrying about having and maintaining their own hardware and software which may result in reduced costs. This allows organisations and groups to act with greater agency to achieve their goals, where agency is defined as "an actor's or group's ability to make purposeful choices - that is, the actor is able to envisage and purposively choose options" (Alsop, Bertelsen and Holland 2006, p.10).

Well-being enhancements are another benefit of cloud computing to African countries. With respect to the health and business sectors, well-being achievements linked to cloud computing may be viewed in terms of social and economic development as well as medical/health wellbeing. Enhancements in business processes can support economic well-being with impacts on other areas. Cloud computing also offers advantages for users which can lead to social and economic wellbeing because hardware and software can be easily provisioned at minimal cost for those without the 
necessary infrastructure. In addition, cloud computing can go a long way to offer health solutions and therefore result in health wellbeing because of possible collaborations between medical practitioners where previously the possibilities were limited or next to none.

Cloud computing offers both infrastructural and provisioning freedoms at the organisational level as well as other knock on effects that enhanced ICT use present at the individual level. By being able to leap frog hardware and software infrastructural provisioning in Africa, cloud computing offers African users the freedom of eliminating potential hardware and software costs through the utilisation of services offered by cloud computing providers and enhancing delivery of business and health services.

Issues of security are a double edged sword. On the one hand, cloud computing brings about security concerns such as management of data in relation to who manages the data and what security measures are in place in order to ensure that users data is secure from potential abuse. However, on the other hand, it might be argued that cloud computing offers potential users the security of knowing they can be able to access cheaper and accessible hardware and software platforms to be able to carry out their business where previously they were unable to do so.

Issues of accountability and responsibility remain unresolved. If we are to look at responsibility as a form of accountability by all stakeholders involved in cloud computing, be it providers or users, this means that all stakeholders ought to play a role in ensuring that cloud computing offers security safe guards and that there is accountability in its provision and its use. This means that issues related to ethical concerns are also adhered to in a responsible fashion. In these ways cloud computing presents opportunities and risks.

To conclude, the highlighted potential and benefits of cloud computing have ethical implications for development linked to the expansion of human, technological and organisational capabilities through resource sharing, cost saving on infrastructure as well as enabling functionings related to process, operations and scaling. The benefits come with ethical drawbacks related to security, privacy, equality as well as dependency on service providers which may lead to questions of unequal power relations. Such drawbacks require urgent attention. One way of addressing the concerns is by having robust policies around identified ethical issues as technologies like cloud computing are further embedded.

\section{Acknowledgements}

The research leading to these results received funding from the European Community's Seventh Framework Programme (FP7/2007-2013) under grant agreements $n^{\circ} 230318$ (ETICA) and from the IDEGOV project funded by the CIGREF foundation. 


\section{References}

Alkire, S. (2008). Using the Capability Approach: Prospective and Evaluative Analyses'. The Capability Approach: Concepts, Measures and Applications, Cambridge University Press, Cambridge, 26-50.Alsop, R., M. Bertelsen, and J. Holland. 2006. “Empowerment in Practice: From Analysis to Implementation." Poverty Reduction Group, World Bank, Washington, DC.

Armbrust, M., Fox, A., Griffith, R., Joseph, A. D., Katz, R., Konwinski, A. \& Zaharia, M. (2010). A view of cloud computing. Communications of the ACM, 53(4), 50-58

Bass, J. M., Nicholson, B., \& Subhramanian, E. (2013). A Framework Using Institutional Analysis and the Capability Approach in ICT4D. Information Technologies \& International Development, 9(1), pp-19.

Bell, M. and Pavitt, K., 1997. Technological accumulation and industrial growth: contrasts between developed and developing countries. Technology, globalisation and economic performance, 83137, pp.83-137.

Britz, J., Ponelis, S. and Lor, P.J., 2013. Distinguishing right from wrong: proposed ethical principles for the development of national information policies. Innovation: journal of appropriate librarianship and information work in Southern Africa, 2013(47), pp.927.

Buyya, R., Yeo, C. S., \& Venugopal, S. (2008). Market-oriented cloud computing: Vision, hype, and reality for delivering it services as computing utilities (pp. 5-13). Presented at the High Performance Computing and Communications, 2008. HPCC'08. 10th IEEE International Conference on, leee.

Capurro, R. (2007a). Information Ethics for and from Africa. International Review of Information Ethics, 7, 1-13. 
Capurro, R. (2007b). Intercultural information ethics. In R. Capurro, J. Frühbauer, \& T. Hausmanninger (Eds.), Localizing the Internet. Ethical Aspects in Intercultural Perspective (Vol. 4, pp. 21-38). Munich: Fink 2007.

Capurro, R. (2008). Intercultural information ethics: foundations and applications. Journal of Information, Communication and Ethics in Society, 6(2), 116-126.

Carroll, M., Van Der Merwe, A., \& Kotze, P. (2011). Secure cloud computing: Benefits, risks and controls (pp. 1-9). Presented at the Information Security South Africa (ISSA), 2011, IEEE.

Clark, D. A. (2002). Development ethics: a research agenda. International Journal of Social Economics, 29(11), 830-848.

Clark, D. A. (2005). The Capability Approach: Its Development, Critiques and Recent Advances.

Coeckelbergh, M. (2011). Human development or human enhancement? A methodological reflection on capabilities and the evaluation of information technologies. Ethics and Information Technology, 13(2), 81-92.

Dahiru, A. A., Bass, J., \& Allison, I. (2014). Cloud Computing: Adoption Issues for Sub-Saharan Africa SMEs. The Electronic Journal of Information Systems in Developing Countries.

Dikaiakos, M. D., Katsaros, D., Mehra, P., Pallis, G., \& Vakali, A. (2009). Cloud computing: distributed internet computing for IT and scientific research. Internet Computing, IEEE, 13(5), 10-13.

Dwivedi, Y. K., \& Mustafee, N. (2010). It's unwritten in the Cloud: the technology enablers for realising the promise of Cloud Computing. Journal of Enterprise Information Management, 23(6), 673-679.

Enos, J. L. (2003). In Pursuit of Science and Technology in Sub-Saharan Africa. Routledge.

Ess, C. (2007). Cybernetic Pluralism in an Emerging Global Information and Computing Ethics. International Review of Information Ethics, 7, 09. 
Estache, A. (2006) Infrastructure: A survey of recent and upcoming issues, accessed from http://siteresources.worldbank.org/INTDECABCTOK2006/Resources/Antonio_Estache_Infr astructure_for_Growth.pdf

ETICA project (2009-2012). (www.etica-project.eu)

Evanoff, R. (2006). Integration in intercultural ethics. International Journal of Intercultural Relations, 30(4), 421-437.

Foster, V., and Briceño-Garmendia, C. (eds.). (2010). Africa's Infrastructure. A Time for Transformation. A co-publication of the Agence Française de Développement and the World Bank.

Gigler, B.-S. (2011). Informational Capabilities-The Missing Link for the Impact of ICT on development. Available at SSRN.

Goonatilake, R., Herath, S., Herath, A., \& Tyska, C. (2009). E-Collaboration Issues in Global Trade, Transactions, and Practices. European Journal of Scientific Research ISSN, 326-336.

Greengard, S. (2010). Cloud computing and developing nations. Communications of the ACM, $53(5), 18-20$.

GSMA (2013). The Mandatory Registration of Prepaid SIM Card Users A White Paper - November 2013, accessed from http://www.gsma.com/publicpolicy/wpcontent/uploads/2013/11/GSMA White-Paper Mandatory-Registration-of-Prepaid-SIMUsers 32pgWEBv3.pdf

Habib, S. M., Hauke, S., Ries, S., \& Mühlhäuser, M. (2012). Trust as a facilitator in cloud computing: a survey. Journal of Cloud Computing: Advances, Systems and Applications, 1(1), 1

Heeks, R. (2009). The ICT4D 2.0 Manifesto: Where Next for ICTs and International Development? Accessed from http://www.oecd.org/dataoecd/43/25/43602651.pdf

Huang, J. and Nicol, D. M. (2013). Trust mechanisms for cloud computing. Journal of Cloud Computing: Advances, Systems and Applications 2.1 (2013): 1 
ITU (2007). Telecommunication/ICT Markets and Trends in Africa 2007. ITU. Accessed from

http://www.itu.int/ITU-D/ict/statistics/material/af report07.pdf

ITU. (2012). Cloud Computing in Africa: Situation and Perspectives. ITU.

ITU (2012). Measuring the Information Society 2012, accessed from

https://www.itu.int/dms_pub/itu-d/opb/ind/D-IND-ICTOI-2012-SUM-PDF-E.pdf

ITU (2015). Measuring the Information Society 2015, accessed from http://www.itu.int/en/ITU-

\section{D/Statistics/Documents/publications/misr2015/MISR2015-w5.pdf}

Johnstone, J. (2007). Technology as empowerment: A capability approach to computer ethics.

Ethics and Information Technology, 9(1), 73-87.

Kizza, J. M. (2009). Building the African ICT Infrastructure for Development: The Role of the African University- Part I. International Journal of Computing and ICT Research, Vol. 3, No. 1, pp. 7-

9.

Kleine, D. (2011). The capability approach and the 'medium of choice': Steps towards conceptualising information and communication technologies for development. Ethics and Information Technology, 13(2), 119-130.

Kshetri, N. (2012). Cloud computing in developing economies. Kshetri, Nir (2010)" Cloud Computing in Developing Economies", IEEE Computer, 43(10), 47-55.

Mansell, Robin (2010). Technology, innovation, power and social consequence. In: Kalantzis-Cope, P. and Martin, K. G., (eds.) Emerging Digital Spaces in Contemporary Society: Properties of Technology. Palgrave Macmillan, New York, USA, pp. 13-25.

Marinos, A., \& Briscoe, G. (2009, December). Community cloud computing. In IEEE International Conference on Cloud Computing (pp. 472-484). Springer Berlin Heidelberg

Matzner, T. (2014). Why privacy is not enough privacy in the context of 'ubiquitous computing' and 'big data'. Journal of Information, Communication and Ethics in Society, 12(2), 93-106.

McCalla, J., Pepper, R., \& Rueda-Sabater, E. (2009). Broadband Across Africa: Accelerating Benefits (Cisco White Paper). Retrieved from http://www.cisco.com/web/about/ac79/docs/Broadband_WP_0831REV_FINAL.pdf 
Mell, P., \& Grance, T. (2011). The NIST definition of cloud computing (draft). NIST Special Publication, 800(145), 7.

Molony, T. (2006). 'I Don't Trust the Phone; It Always Lies': Trust and Information and Communication Technologies in Tanzanian Micro-and Small Enterprises. Information Technologies \& International Development, 3(4), pp-67.

Moor, J. H. (1997). Towards a Theory of Privacy I1', in the Information Age. Computers and Society, $27(3), 27-32$.

Mthoko, H. L., \& Pade-Khene, C. (2013). Towards a theoretical framework on ethical practice in ICT4D programmes. Information Development, 29(1), 36-53.

Mukherjee, A., \& Nath, P. (2007). Role of electronic trust in online retailing: a re-examination of the commitment-trust theory. European Journal of Marketing, 41(9/10), 1173-1202.

Nkosi, M. T. \& Mekuria, F. (2010). Cloud Computing for Enhanced Mobile Health Applications. Cloud Computing Technology and Science (CloudCom), 2010 IEEE Second International Conference on Cloud Computing Technology and Science

Nussbaum, M. (2001). Women and human development: The capabilities approach (Vol. 3). Cambridge University Press.

Quinn, M. J. (2006). (2nd Ed) Ethics for the Information Age. Pearson International Edition Ratten, V. (2012). Entrepreneurial and ethical adoption behaviour of cloud computing. The Journal of High Technology Management Research Vol 23, Issue 2, 2012, pp. 155-164

Reidl, C., Wagner, I., \& Rauhala, M. (n.d.). Examining Ethical Issues of IT in Health Care.

Robeyns, I. (2005). The capability approach: a theoretical survey. Journal of Human Development, 6(1), 93-117.

Samuel, O., Omisore, M., Ojokoh, B., \& Atajeromavwo, E. (2013). Enhanced cloud based model for healthcare delivery organizations in developing countries. Int J Comput Appl, 74, 09758887. 
Sen, A. (2001). Development as Freedom. Oxford and New York: Oxford University Press.

Sen, A. (2009). The Idea of Justice. Harvard University Press.

Senanu, D. R., Ikhu-Omoregbe, N., \& Ayo Charles, K. (2010). E-Health Application Virtualisation for Nigerian Teaching Hospitals: A Cloud Computing Approach. In international Conference on Cloud Computing \& Virtualisation (pp. 16-19). Singapore.

Smith, M. L., Spence, R., \& Rashid, A. T. (2011). Mobile phones and expanding human capabilities. Information Technologies \& International Development, 7(3), pp-77.

Stahl, B. C., Eden, G., Jirotka, M., \& Coeckelbergh, M. (2014). From Computer Ethics to Responsible Research and Innovation in ICT: The transition of reference discourses informing ethicsrelated research in information systems. Information \& Management.

Stahl, B. C.; Heersmink, R; Goujon, P., Flick, C.; van den Hoven, J. \& Wakunuma, K. (2010). Identifying the Ethics of Emerging Information and Communication Technologies: An Essay on Issues, Concepts and Method In: International Journal of Technoethics. Pp. 20-38

Subashini, S., \& Kavitha, V. (2011). A survey on security issues in service delivery models of cloud computing. Journal of Network and Computer Applications, 34(1), 1-11.

Székely, I., Dániel Szabó, M., \& Vissy, B. (2011). Regulating the future? Law, ethics, and emerging technologies. Journal of Information, Communication and Ethics in Society, 9(3), 180-194.

Timmermans, J., Stahl, B., Ikonen, V. \& Bozdag, E (2010) The Ethics of Cloud Computing: A Conceptual Review. Cloud Computing Technology and Science (CloudCom), 2010 IEEE Second International Conference on Cloud Computing Technology and Science UNCTAD (2013). Informatlon Economy Report: The Cloud Economy and Developing Countries. United Nations

UNCTAD (2013). World Investment Report Global Value Chains: Investment and trade for Development 2013, accessed from http://unctad.org/en/PublicationsLibrary/wir2013_en.pdf 
Wakunuma, K. and Stahl, B.C. (2014). Tomorrow's ethics and today's response: An investigation into the ways information systems professionals perceive and address emerging ethical issues. Information Systems Frontiers, (2014) 16: 383-397

Walsham, G. (2006). Doing interpretive research. European Journal of Information Systems June 2006, Volume 15, Issue 3, pp 320-330

Wangwe, S. M., \& Semboja, H. H. (2003). Impact of structural adjustment on industrialization and technology in Africa. African Voices on Structural Adjustment, 161.

Weber, A. S. (2011). Cloud Computing in Education in the Middle East and North Africa (MENA) Region: Can barriers be overcome? Proceedings of the $7^{\text {th }}$ International Scientific Conference: eLearning and Software for Education (eLSE), pp. 565-570

Williams, M. D. (2010). Broadband for Africa: developing backbone communications networks. World Bank Publications.

Williams, M. D. J.; Mayer, R. \& Minges, M. (2011). Africa's ICT Infrastructure: Building on the Mobile Revolution, accessed from http://siteresources.worldbank.org/INFORMATIONANDCOMMUNICATIONANDTECHNOLO GIES/Resources/AfricasICTInfrastructure_Building_on_MobileRevolution_2011.pdf

Zheng, Y., \& Stahl, B. C. (2011). Technology, capabilities and critical perspectives: what can critical theory contribute to Sen's capability approach? Ethics and Information Technology, 13(2), $69-80$.

Zissis, D. \& Lekkas, D. (2010). Addressing cloud computing security issues. Future Generation Computer Systems. Vol 28, Issue 3, March 2012, pp. 583-592

\section{Appendix A: Survey and Interview schedule}

Survey schedule - Respondent Information

1. Gender: 
2. Position in organisation:

3. Location e.g. Africa, Asia, Europe, and America:

4. Age:

5. Education background:

Interview Questions on Emerging Technologies and associated ethical issues

1. Is your organisation currently looking to develop or be involved in any emerging technologies?

a. If Yes, can you give a list of what these emerging technologies will be?

b. And if Yes, why has your organisation chosen the listed emerging technologies?

2. What application areas will they be used for?

3. In what way do you think the listed technologies are emerging?

4. What impact do you think the emerging technologies will have in the area of application?

5. What ethical issues do you think they might produce?

6. Why do you think these might be ethical issues?

7. By what means do you foresee them to be ethical issues?

8. Are there any measures that have been put in place in order to overcome the foreseeable ethical issues? 